\title{
Effect of long-term GH replacement therapy on cardiovascular outcomes in isolated GH deficiency compared with multiple pituitary hormone deficiencies: a sub-analysis from the Dutch National Registry of Growth Hormone Treatment in Adults
}

\author{
Christa C van Bunderen ${ }^{1}$, Carline J van den Dries', Martijn W Heymans ${ }^{2}$, \\ Anton A M Franken ${ }^{3}$, Hans P F Koppeschaar ${ }^{4}$, Aart J van der Lely ${ }^{5}$ and \\ Madeleine L Drent ${ }^{1}$ \\ ${ }^{1}$ Section of Endocrinology, Department of Internal Medicine, Neuroscience Campus Amsterdam and ${ }^{2}$ Department \\ of Epidemiology and Biostatistics, VU University Medical Center, De Boelelaan 1117, 1081 HV Amsterdam, \\ The Netherlands, ${ }^{3}$ Department of Internal Medicine, Isala Clinics, Zwolle, The Netherlands, ${ }^{4}$ Emotional Brain and \\ Alan Turing Institute for Multidisciplinary Health Research, Almere, The Netherlands and ${ }^{5}$ Division of Endocrinology \\ and Metabolism, Department of Internal Medicine, Erasmus Medical Center, Rotterdam, The Netherlands
}

Correspondence
should be addressed
to C C van Bunderen
Email
c.vanbunderen@vumc.nl

\begin{abstract}
Objective: Isolated GH deficiency (IGHD) could provide a model to investigate the influence of GH deficiency per se and the effect of $\mathrm{GH}$ replacement therapy without the influence from other pituitary hormone deficiencies or their treatment. The aim of this study is to address the questions about differences between IGHD and multiple pituitary hormone deficiencies (MPHDs) in clinical presentation and in responsiveness to $\mathrm{GH}$ treatment.

Design: A nationwide surveillance study was carried out to describe the difference in the clinical presentation and responsiveness to GH treatment of patients with IGHD and MPHDs.

Methods: The Dutch National Registry of GH Treatment in Adults was founded in 1998 to gain more insight into long-term efficacy and safety of GH therapy. Out of 2891 enrolled patients, 266 patients with IGHD at the start of GH treatment were identified and compared with 310 patients with MPHDs. Cardiovascular indices will be investigated at baseline and during long-term follow-up, including body composition, lipid profile, glucose metabolism, blood pressure, and morbidity.

Results: Patients with IGHD and MPHDs were demonstrated to be different entities at clinical presentation. Metabolically, patients with MPHDs had a larger waist circumference, lower HDL cholesterol level, and higher triglyceride level. The effect of GH treatment was comparable between patient groups. GH seems to protect against rising lipid levels and blood pressure, even after excluding patients using corresponding concomitant medication. The risk for cardiovascular disease or diabetes mellitus during follow-up was not different between patients with IGHD and MPHDs.

Conclusions: Patients with IGHD had a less impaired metabolic profile than patients with MPHDs at baseline. Influence of other pituitary hormone replacement therapies on the effect of GH treatment is not demonstrated.
\end{abstract}




\section{Introduction}

Growth hormone deficiency (GHD) in adults has become increasingly recognized as an important (metabolic) syndrome (1). Significant changes in cardiovascular risk factors, such as an adverse lipid profile, altered body composition associated with an increased fat mass and BMI, and hypertension, are reported $(1,2)$. Adults with GHD have a decreased life expectancy due to an increased mortality from cardiovascular diseases (CVDs) $(3,4)$. Many studies have demonstrated the positive effects of GH replacement therapy on cardiovascular risk factors in adults with $\operatorname{GHD}(5,6)$, even up to a follow-up of 10 years $(7,8)$. Nevertheless, only a few studies have reported on the effect of GH on cardiovascular morbidity (9, 10, 11). Holmer et al. (10) described a decreased incidence of cardiac events during GH therapy, especially in men, compared with a control cohort. They conclude a protective role of $\mathrm{GH}$, possibly in combination with more hospital visits and subsequently earlier start of cardioprotective medication. Data on mortality in GH-treated adults is also still scarce (12). In an earlier study from the Dutch National Registry of Growth Hormone Treatment in Adults, we demonstrated that, in 2229 patients after 13.353 years of treatment, the standardized mortality rate was found to decrease to normal in men and to remain increased in women, especially due to CVD (13). Gender difference in the effect of GH treatment has been described several times $(14,15,16,17)$. The explanation is often looked for in other pituitary hormone axes. The influence of other pituitary hormone deficiencies or their replacement in combination with GH treatment on CVDs merits more detailed investigations. Theoretically, isolated GH deficiency (IGHD) provides a model to characterize the influence of GHD per se, without the influence from other pituitary hormone deficiencies or their treatment. In patients with GHD, the prevalence of IGHD is reported to be $8-16 \%(18,19,20)$, and therefore, large cohorts are required.

In this nationwide surveillance study, the Dutch National Registry of Growth Hormone Treatment in Adults, we describe the difference in clinical presentation between patients with IGHD and those with multiple pituitary hormone deficiencies (MPHDs) at the start of GH treatment. Subsequently, we compare the long-term effect of GH treatment on cardiovascular risk factors and morbidity in patients with IGHD and MPHDs taking into account the use of cardioprotective medication and evolution of pituitary hormone deficiencies over time.

\section{Subjects and methods}

\section{Study population}

The Dutch National Registry of Growth Hormone Treatment in Adults was established in 1998 as an initiative of the Ministry of Health to gain insight into long-term efficacy, safety, and costs of GH treatment in The Netherlands. Approval by an independent board of endocrinologists was required for the reimbursement of expenses for GH treatment by the health insurer, and a registry was linked to this assessment. Severe GHD was defined according to the consensus guidelines of the Growth Hormone Research Society (21). Until 2009, a total of 2891 patients were registered. Their characteristics and diagnostic test procedures were described in more detail previously (20). Of the patients who were diagnosed with GHD in childhood, 95\% have been treated with GH during childhood before registration. They were retested after at least three months of GH withdrawal.

Based on the recorded deficiencies at registration as diagnosed by the attending endocrinologist and checked by usage of pituitary hormone substitution therapies, 311 patients were identified as having IGHD at the start of GH treatment. Accordingly, a random subset of patients with MPHDs $(n=318)$ was selected from the database, with the aim to represent clinical practice. Patients who had $<30$ days of available follow-up data were excluded for analyses resulting in 266 and 310 patients for analysis respectively (Fig. 1). The selected subset of patients with MPHDs differed minimally from the rest of the patients with MPHDs in the cohort, but demonstrated more GHD of childhood onset (26 vs $20 \%, P=0.01$ ) and a slightly lower BMI (26.9 vs 27.7 , $P=0.04$ ). Of the subset of patients with MPHDs, $86.5 \%$ demonstrated secondary hypogonadism, $85.5 \%$ secondary hypothyroidism, $76.5 \%$ secondary hypocortisolism, and 22.9\% ADH deficiency at baseline. Patients lost to followup, who died, or who stopped GH treatment were censored in the analysis. The reason for discontinuation of GH was related to CVD or the patient's metabolic profile in only two of the 36 cases. During GH treatment, 23 patients died (4\%), including six patients with IGHD (2.3\%) and 17 patients with MPHDs (5.5\%), where CVDs as the cause of death was equally divided with 40 and $36 \%$ respectively.

\section{Measurements}

Data were collected (bi-)annually from medical records by trained monitors. As an internal quality control, in $10 \%$ of 


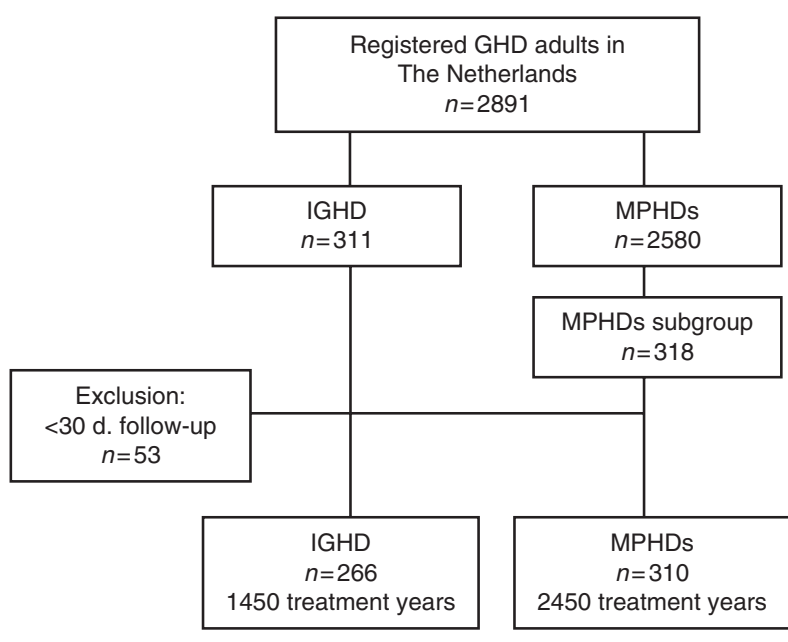

\section{Figure 1}

Design of study sample. GHD, growth hormone deficiency; IGHD, isolated growth hormone deficiency; MPHDs, multiple pituitary hormone deficiencies; $\mathrm{GH}$, growth hormone.

patients, data were collected twice by different monitors. Person-years of treatment were calculated from the date of starting GH treatment in adulthood (baseline) until the last date of follow-up, discontinuation of $\mathrm{GH}$, or death. Underlying diagnoses for GHD were categorized into five groups (GH-/adrenocorticotropin (ACTH)-secreting adenoma, non-functioning pituitary adenoma, craniopharyngioma, possible malignant causes, and others including prolactinoma, congenital, Sheehan's syndrome, trauma, etc.), as described in more detail previously (13). The dose of $\mathrm{GH}$ treatment was individualized by the attending physician with the common aim of normalizing insulin-like growth factor 1 (IGF1) SDS for age and gender. Statin, antihypertensive, antidiabetic, and antiplatelet medication use was recorded at baseline and during follow-up. Data on cardiovascular risk factors included in our analyses were height and weight to calculate the BMI $\left(\mathrm{kg} / \mathrm{m}^{2}\right)$, waist circumference $(\mathrm{cm})$, systolic, and diastolic blood pressure (mmHg). Measurements of total, HDL, and LDL cholesterol, triglycerides (all expressed as mmol/l), and HbA1c (\%) were performed locally by routine laboratory techniques. Medical history and adverse events were recorded thoroughly and (at least) coded for cardiacrelated diseases (e.g. myocardial infarction, heart failure, and coronary interventions), peripheral vascular diseases (e.g. atherosclerosis, intermittent claudication, and vascular surgery), stroke, and diabetes mellitus, the latter verified by medication use. Date and cause of death were collected through medical records and death certification from the Dutch Central Bureau of Statistics, as described in more detail previously (13). Evolving additional pituitary deficiencies over time were identified by the start of the corresponding substitution therapies, based on diagnostic tests performed by the discretion of the attending endocrinologist.

\section{Statistical analyses}

Categorical data are expressed as numbers (\%) and continuous data as mean (s.D.), or as median (interquartile range $(\mathrm{IQR})$ ) for skewed variables. Parametric or nonparametric tests were used when appropriate. Two-sided $P \leq 0.05$ were considered significant. Statistical analyses were performed using the statistical software package IBM SPSS statistics 20.0 (SPSS, Inc.) and SAS 9.2 (SAS Institute, Inc., Cary, NC, USA). To estimate changes in the outcome measures over time, linear mixed models (LMMs) were used and time was included in these models as a continuous variable, i.e. the time points were different for each patient. Mixed models use all available data, properly accounting for the correlation between repeated measurements within patients over time. These models can also properly deal with missing data by randomly assuming the details; the same was followed for our missing data on cardiovascular risk factors. As recommended by Fitzmaurice et al. (22), we included the baseline values of risk factors in our LMMs as part of the outcome variable, to correctly handle missing baseline values. To evaluate the need for baseline adjustment, we conducted several sensitivity analyses and included the baseline value of each outcome variable as a covariate in our LMMs after we applied multiple imputation $(m=20)$ to substitute the missing baseline values. These sensitivity analyses did not alter the results and, therefore, we used the LMMs with baseline values as part of the outcome variable similar to our primary data analyses. To study the increase or decrease in effect between the group of patients with IGHD and the group of patients with MPHDs over time, interaction terms between time and group were introduced in all models. The assumptions of the LMMs were checked by using residual plots and by evaluating the residual variance over time. Analyses were conducted unadjusted, adjusted for age and sex, and stratified by sex. Sensitivity analyses were conducted to account for the use of cardioprotective medication and evolution from IGHD to MPHDs. To investigate the influence of the inevitable heterogeneity between the groups, we repeated the analyses after selecting only adult-onset GHD and only patients with a non-functioning pituitary adenoma as an underlying cause of GHD. A Cox proportional hazard 
Table 1 Baseline characteristics of patients with IGHD and MPHDs. Data are expressed as numbers (\%) unless specified otherwise.

\begin{tabular}{|c|c|c|c|c|}
\hline & IGHD & MPHDs & $\begin{array}{c}P \\
\text { value }^{a}\end{array}$ & $\begin{array}{c}P \\
\text { value }^{b}\end{array}$ \\
\hline No. of patients & 266 & 310 & & \\
\hline Age, year (S.D.) & $35.1(15.5)$ & $42.5(16.7)$ & $<0.001$ & \\
\hline Sex, no. of females & $143(53.8)$ & $149(48.1)$ & 0.17 & \\
\hline Onset of GHD, CO & $102(38.3)$ & $80(25.8)$ & 0.001 & \\
\hline \multicolumn{5}{|l|}{ Underlying diagnosis } \\
\hline Others & $129(48.5)$ & $119(38.4)$ & 0.02 & \\
\hline $\begin{array}{l}\text { Non-secreting } \\
\text { adenoma }\end{array}$ & $34(12.8)$ & $98(31.6)$ & $<0.001$ & \\
\hline $\begin{array}{l}\text { Secreting adenoma } \\
(\mathrm{GH}, \mathrm{ACTH})\end{array}$ & $29(10.9)$ & $32(10.3)$ & 0.82 & \\
\hline Craniopharyngioma & $3(1.1)$ & 43 (13.9) & $<0.001$ & \\
\hline $\begin{array}{l}\text { Possible malignant } \\
\text { causes }\end{array}$ & $71(26.7)$ & $18(5.8)$ & $<0.001$ & \\
\hline Cranial radiotherapy & $116(43.8)$ & $129(41.9)$ & 0.65 & \\
\hline Pituitary surgery & $75(28.2)$ & $183(59.0)$ & $<0.001$ & \\
\hline \multicolumn{5}{|l|}{ Smoking } \\
\hline Yes & $46(27.7)$ & 77 (29.4) & 0.71 & \\
\hline Former & $16(9.6)$ & $43(16.4)$ & 0.05 & \\
\hline No & $104(62.7)$ & $142(54.2)$ & 0.09 & \\
\hline Alcohol use & $51(36.4)$ & $85(40.7)$ & 0.09 & \\
\hline \multicolumn{5}{|l|}{ Medical history } \\
\hline Cardiac disease & $16(6.1)$ & $25(8.3)$ & 0.31 & 0.45 \\
\hline $\begin{array}{l}\text { Peripheral vascular } \\
\text { disease }\end{array}$ & $5(1.9)$ & $6(2.0)$ & 0.93 & 0.38 \\
\hline Stroke & $3(1.1)$ & $12(4.0)$ & 0.04 & 0.15 \\
\hline Diabetes mellitus & $14(5.3)$ & $20(6.7)$ & 0.50 & 0.62 \\
\hline
\end{tabular}

IGHD, isolated growth hormone deficiency; MPHDs, multiple pituitary hormone deficiencies; $\mathrm{CO}$, childhood onset.

${ }^{a}$ Continuous variables were compared by Student's $t$-test and categorical variables by the $\chi^{2}$-test.

${ }^{b}$ Adjusted for age.

model was used to evaluate the risk of developing CVDs (including stroke) and diabetes mellitus. Two models were applied to adjust for potential confounders. Potential confounders (underlying diagnosis, pituitary surgery, cranial radiotherapy, smoking habits, alcohol use, and BMI) were tested and, in case of more than $10 \%$ change in regression coefficient, included in the fully adjusted model together with age and sex. The individual variables were checked for multicollinearity and linearity. The proportional hazard model assumption was not violated in any of the models.

\section{Results}

\section{Diagnostic procedures}

In $94.7 \%$ of patients with IGHD, the diagnosis of severe GHD was based on a stimulation test $(66.7 \%$ insulin tolerance test (ITT), 15.5\% GHRH/arginine, 7.9\% GHRH,
$8.7 \%$ arginine, and $1.2 \%$ other) with a peak GH response below the threshold for the specific stimulation test in combination with a serum IGF1 level $\leq-2$ s.D. for age and gender or a second stimulation test. This was the case in $89.7 \%$ of patients with MPHDs (41.4\% ITT, $19.8 \%$ arginine, $19.4 \% \mathrm{GHRH} /$ arginine, $17.6 \% \mathrm{GHRH}$, and $1.8 \%$ other). The remaining patients had either a serum IGF1 level $\leq-2$ s.D. with two or more additional pituitary hormone deficits or an evident hypothalamic-pituitary disease where retesting was considered unnecessary (1\%). Patients with IGHD had a median peak GH response of $1.3 \mu \mathrm{g} / \mathrm{l}$ (IQR 1.8) and those with MPHDs had a median peak GH response of $0.6 \mu \mathrm{g} / \mathrm{l}(\mathrm{IQR} 1.1)(P<0.001)$.

\section{Baseline characteristics}

Patient characteristics of both the groups with IGHD and MPHDs are shown in Table 1 . With respect to the metabolic profile, there were significant differences detrimental to patients with MPHDs. After adjustment for age, HDL cholesterol (in males) and triglycerides remained significantly different (Table 2). There was no significant

Table 2 Baseline characteristics on cardiovascular risk factors of patients with IGHD and MPHDs. Data are expressed as mean (s.D.) unless specified otherwise. Waist circumference and HDL cholesterol are demonstrated as stratified by gender suitable for gender-specific normal values.

\begin{tabular}{|c|c|c|c|c|}
\hline & IGHD & MPHDs & $\begin{array}{c}P \\
\text { value }^{a}\end{array}$ & $\begin{array}{c}P \\
\text { value }^{b}\end{array}$ \\
\hline Height (m) & $1.67(0.11)$ & $1.70(0.10)$ & $<0.001$ & $<0.001$ \\
\hline BMI $\left(\mathrm{kg} / \mathrm{m}^{2}\right)$ & $27.1(6.6)$ & $27.5(5.8)$ & 0.50 & 0.56 \\
\hline \multicolumn{5}{|c|}{ Waist circumference $(\mathrm{cm})$} \\
\hline Female & $90(16)$ & $94(13)$ & 0.13 & 0.83 \\
\hline Male & $92(14)$ & $100(13)$ & 0.001 & 0.05 \\
\hline $\begin{array}{l}\text { Total cholesterol } \\
(\mathrm{mmol} / \mathrm{l})\end{array}$ & $5.39(1.14)$ & $5.68(1.15)$ & 0.01 & 0.22 \\
\hline \multicolumn{5}{|c|}{ HDL cholesterol (mmol/l) } \\
\hline Female & $1.38(0.40)$ & $1.36(0.47)$ & 0.82 & 0.78 \\
\hline Male & $1.22(0.30)$ & $1.06(0.33)$ & 0.001 & 0.001 \\
\hline $\begin{array}{l}\text { LDL cholesterol } \\
(\mathrm{mmol} / \mathrm{l})\end{array}$ & $3.35(0.87)$ & $3.54(1.06)$ & 0.15 & 0.62 \\
\hline $\begin{array}{l}\text { Triglycerides } \\
(\mathrm{mmol} / \mathrm{l})^{c}\end{array}$ & $1.26(1.00)$ & $1.79(1.32)$ & $<0.001$ & $<0.001$ \\
\hline HbA1c $(\%)$ & $5.4(0.8)$ & $5.4(0.9)$ & 0.75 & 0.48 \\
\hline $\begin{array}{l}\text { Systolic blood } \\
\text { pressure }(\mathrm{mmHq})\end{array}$ & $125(20)$ & $130(19)$ & 0.02 & 0.86 \\
\hline $\begin{array}{l}\text { Diastolic blood } \\
\text { pressure }(\mathrm{mmHg})\end{array}$ & $77(12)$ & $80(11)$ & 0.002 & 0.26 \\
\hline
\end{tabular}

IGHD, isolated growth hormone deficiency; MPHDs, multiple pituitary hormone deficiencies.

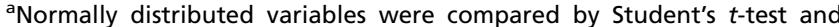
skewed variables by the Mann-Whitney $U$ test.

${ }^{\mathrm{b}}$ Adjusted for age.

${ }^{\mathrm{C}}$ Median (IQR). 
difference in use of cardioprotective medication between the groups at baseline. When investigating patients with only adult-onset GHD or a non-functioning pituitary adenoma as the underlying cause of GHD, the difference in waist circumference equalized, but the differences in lipid profile remained (data not shown).

\section{Long-term follow-up}

The median follow-up of patients with IGHD was 4.9 years (IQR 5.9), representing 1450 years of treatment. For patients with MPHDs, the median follow-up was 8.4 years (IQR 2.3), representing 2450 years of treatment. During follow-up, male patients with IGHD were administered a daily median GH dose of $0.37 \mathrm{mg}$ (IQR 0.24) and those with MPHDs received $0.34 \mathrm{mg}$ (IQR 0.25) $(P=0.25$, adjusted for age). In female patients with IGHD and MPHDs, the daily median GH dose was $0.37 \mathrm{mg}$ (IQR 0.35) and $0.48 \mathrm{mg}$ (IQR 0.30) respectively. After adjusting for age and usage of oral estrogens, there was still a significant difference $(P=0.02)$. During follow-up, antihypertensive drugs were used by $22.6 \%$ of patients with IGHD compared with $30.4 \%$ in the MPHD group $(P=0.03)$. Use of statin was reported in $14.7 \%$ of patients with IGHD compared with $27.8 \%$ in the MPHD group $(P<0.001)$. Antidiabetic drugs were used by $5.6 \%$ of patients with IGHD and $11 \%$ of patients with MPHDs $(P=0.02)$.

\section{Effect of long-term GH treatment on cardiovascular risk factors}

The estimates of changes over time for both groups of patients separately, and the interaction between time and group resembling the difference in effect between the groups, are given in Table 3. Adjustment for age and gender did not significantly change any of the results. A significant increase in the BMI and waist circumference is demonstrated in both groups, which is significantly higher in patients with IGHD compared with those having MPHDs. After stratification for gender, the difference in BMI between patients with IGHD and those with MPHDs was more prominent in men (estimation $0.21,95 \%$ CI 0.10 to $0.32, P<0.001$; representing a $0.21 \mathrm{~kg} / \mathrm{m}^{2}$ per year steeper increase in IGHD compared with MPHDs) and not significant in women (estimation $0.09,95 \%$ CI -0.04 to $0.22, P=0.18$ ). For waist circumference, the difference between groups was also more prominent in men (estimation $0.84,95 \%$ CI 0.44 to $1.23, P<0.001$ ), whereas not significant in women (estimation $0.33,95 \%$ CI -0.19 to $0.86, P=0.21$ ). The lipid profile improved significantly in both groups, except for triglyceride level in patients with IGHD. Patients with MPHDs had a better improvement than patients with IGHD, except for HDL cholesterol, which increased equally. After stratification for gender, the difference in triglyceride level was more prominent in women (estimation $0.05,95 \%$ CI 0.01 to $0.09, P=0.01$ ), whereas it was not significant in men (estimation 0.02, $95 \%$ CI -0.02 to $0.07, P=0.34$ ).

Excluding patients using statins resulted in loss of effect on total cholesterol in patients with IGHD (estimation $-0.01,95 \% \mathrm{CI}-0.03$ to $0.01, P=0.41$ ) and on LDL cholesterol (estimation $-0.02,95 \% \mathrm{CI}-0.04$ to 0.01 , $P=0.16$ ), and for both, the significant difference when compared with patients with MPHDs disappeared. The positive effect on HDL cholesterol persisted, as did the difference in effect between the groups for triglycerides. Excluding patients using antidiabetic medication did not change the results for HbA1c. Excluding patients who used

Table 3 Estimates of changes over time analyzed by linear mixed models for patients with IGHD and MPHDs. Values are estimates of changes over time per year $(95 \% \mathrm{Cl})$.

\begin{tabular}{|c|c|c|c|c|c|c|}
\hline & \multicolumn{2}{|l|}{ IGHD } & \multicolumn{2}{|l|}{ MPHDs } & \multicolumn{2}{|l|}{ IGHD vs MPHDs } \\
\hline & Estimate $(95 \% \mathrm{Cl})$ & $P$ value & Estimate $(95 \% \mathrm{Cl})$ & $P$ value & Estimate $(95 \% \mathrm{Cl})^{\mathrm{a}}$ & $P$ value \\
\hline BMI $\left(\mathrm{kg} / \mathrm{m}^{2}\right)$ & $0.28(0.21$ to 0.35$)$ & $<0.001$ & $0.13(0.08$ to 0.18$)$ & $<0.001$ & 0.14 (0.06 to 0.23$)$ & 0.001 \\
\hline Waist circumference $(\mathrm{cm})$ & $0.88(0.63$ to 1.14$)$ & $<0.001$ & $0.28(0.08$ to 0.47$)$ & 0.006 & 0.61 (0.29 to 0.93$)$ & $<0.001$ \\
\hline Total cholesterol (mmol/l) & $-0.03(-0.05$ to -0.01$)$ & 0.02 & $-0.08(-0.10$ to -0.06$)$ & $<0.001$ & $0.05(0.02$ to 0.08$)$ & 0.001 \\
\hline HDL cholesterol (mmol/l) & $0.02(0.01$ to 0.02$)$ & $<0.001$ & 0.01 (0.01 to 0.02$)$ & $<0.001$ & $0.002(-0.01$ to 0.01$)$ & 0.69 \\
\hline LDL cholesterol (mmol/l) & $-0.03(-0.06$ to -0.01$)$ & 0.01 & $-0.07(-0.09$ to -0.05$)$ & $<0.001$ & 0.04 (0.004 to 0.07$)$ & 0.03 \\
\hline Triglycerides (mmol/l) & $0(-0.02$ to 0.03$)$ & 0.87 & $-0.04(-0.05$ to -0.02$)$ & $<0.001$ & 0.04 (0.01 to 0.07$)$ & 0.02 \\
\hline $\mathrm{HbA1c}(\%)$ & 0.03 (0.01 to 0.04$)$ & 0.004 & $0.03(0.01$ to 0.04$)$ & $<0.001-$ & $-0.003(-0.02$ to 0.02$)$ & 0.81 \\
\hline Systolic blood pressure $(\mathrm{mmHg})$ & $-0.14(-0.48$ to 0.19$)$ & 0.41 & $-0.12(-0.36$ to 0.11$)$ & 0.32 & $-0.02(-0.43$ to 0.39$)$ & 0.93 \\
\hline Diastolic blood pressure $(\mathrm{mmHg})$ & $0.10(-0.10$ to 0.30$)$ & 0.34 & $-0.17(-0.32$ to -0.03$)$ & 0.02 & 0.27 (0.02 to 0.52$)$ & 0.03 \\
\hline
\end{tabular}

IGHD, isolated growth hormone deficiency; MPHDs, multiple pituitary hormone deficiencies.

${ }^{a}$ Interaction term between time and group to estimate the difference in effect over time. 
Table 4 Hazard ratio for developing cardiovascular disease and diabetes mellitus in patients with IGHD compared with patients having MPHDs. Adjusted: sex and age. Fully adjusted: sex, age, pituitary surgery, and cranial radiotherapy (cardiovascular disease). Sex, age, pituitary surgery, and body mass index (diabetes mellitus).

\begin{tabular}{|c|c|c|}
\hline & HR $(95 \% \mathrm{Cl})$ & $P$ value \\
\hline \multicolumn{3}{|l|}{ Cardiovascular disease } \\
\hline Unadjusted model & $0.71(0.29-1.73)$ & 0.45 \\
\hline Adjusted model & $1.05(0.42-2.63)$ & 0.91 \\
\hline Fully adjusted model & $1.07(0.42-2.69)$ & 0.89 \\
\hline \multicolumn{3}{|l|}{ Diabetes mellitus } \\
\hline Unadjusted model & $0.58(0.23-1.47)$ & 0.25 \\
\hline Adjusted model & $0.81(0.31-2.12)$ & 0.67 \\
\hline Fully adjusted model & $1.22(0.45-3.29)$ & 0.69 \\
\hline
\end{tabular}

IGHD, isolated growth hormone deficiency; MPHDs, multiple pituitary hormone deficiencies; HR, hazard ratio.

antihypertensive drugs during follow-up resulted in an increase in diastolic blood pressure of $0.22 \mathrm{mmHg}$ yearly in patients with IGHD (95\% CI -0.00 to $0.44, P=0.05$ ). The difference in effect over time when compared with patients with MPHDs decreased and lost significance. When investigating only patients with adult-onset GHD, and excluding patients using concomitant medication, very few relevant changes in the results were noticed. Despite the loss of power on estimates of changes in some variables, the difference in effect on total cholesterol levels between the groups alone gained significance (estimation $0.04,95 \%$ CI 0.003 to $0.07, P=0.03$ ). When investigating only patients with a non-functioning pituitary adenoma as an underlying cause of GHD, the difference in increase in the BMI and waist circumference between patients with IGHD and those with MPHDs disappeared (BMI: estimation $0.01,95 \%$ CI -0.16 to $0.17, P=0.90$; waist circumference: estimation $0.10,95 \%$ CI -0.79 to 0.98 , $P=0.83$ )

\section{Effect of long-term GH treatment on morbidity}

After excluding patients with a medical history of CVDs and those with missing data on adverse events $(n=5)$ in the group of patients, 237 with IGHD and 261 with MPHDs were included in the analyses. In total, 24 cardiovascular events were observed during follow-up (seven in IGHD and 17 in MPHD). Most of the total number of events $(n=16)$ included cardiac-related disorders, followed by seven cases of stroke and one peripheral vascular disease. The risk of developing CVDs was not significantly different for patients with IGHD when compared with patients with MPHDs (Table 4). After excluding patients with prevalent diabetes mellitus at baseline and patients with missing data on adverse events, 244 patients with IGHD and 280 patients with MPHDs were included in the analysis. Of them, eight patients with IGHD and 18 patients with MPHDs developed diabetes. The risk for patients with IGHD was not significantly different when compared with patients with MPHDs (Table 4).

\section{Developing new pituitary deficiencies over time}

During follow-up, 45 patients (17\%) with IGHD developed a new pituitary deficiency, compared with 37 patients (12\%) with MPHDs. The risk of developing an additional pituitary deficiency was almost two times higher in patients with IGHD compared with those with MPHDs (hazard ratio (HR) 1.90, 95\% CI 1.23-2.95, $P=0.004$ ). The numbers of new deficiencies in addition to the number of deficiencies at baseline are given in Table 5. New deficiencies were diagnosed even up to 10 years after baseline. As it represents clinical practice and the evolution from IGHD to MPHDs occurred after 43\% of the total follow-up duration, these 45 patients were

Table 5 New pituitary hormone deficiencies additional to the number of pituitary hormone deficiencies at baseline (in addition to growth hormone). Data are expressed as numbers (\%).

\begin{tabular}{|c|c|c|c|c|c|}
\hline \multirow[b]{2}{*}{ Baseline } & \multicolumn{4}{|c|}{ New pituitary hormone deficiency } & \multirow[b]{2}{*}{ Total } \\
\hline & 0 & 1 & 2 & 3 & \\
\hline \multicolumn{6}{|l|}{ MPHDs } \\
\hline 1 & 31 (73.8) & 5 (11.9) & 6 (14.3) & $0(0.0)$ & $42(100)$ \\
\hline 2 & 41 (70.7) & $17(29.3)$ & $0(0.0)$ & $0(0.0)$ & $58(100)$ \\
\hline 3 & $146(94.2)$ & $9(5.8)$ & $0(0.0)$ & $0(0.0)$ & 155 (100) \\
\hline 4 & $55(100)$ & $0(0.0)$ & $0(0.0)$ & $0(0.0)$ & 55 (100) \\
\hline Total & $273(88.1)$ & $31(10.0)$ & $6(1.9)$ & $0(0.0)$ & $310(100)$ \\
\hline \multicolumn{6}{|l|}{ IGHD } \\
\hline 0 & 221 (83.1) & $32(12.0)$ & $9(3.4)$ & $4(1.5)$ & $266(100)$ \\
\hline
\end{tabular}

IGHD, isolated growth hormone deficiency; MPHDs, multiple pituitary hormone deficiencies. 
analyzed as belonging to the IGHD group. A sensitivity analysis demonstrated that analyzing these patients as having MPHDs instead of IGHD did not significantly change the results.

\section{Discussion}

Isolated GHD provides a model to characterize the influence of GHD and GH treatment without the influence from other pituitary hormone deficiencies or their replacement therapies. We aimed to address the questions about differences between patients with IGHD and those with MPHDs in clinical presentation and in responsiveness to GH replacement therapy with respect to cardiovascular risk factors and morbidity. Owing to the observational nature of this national registry, we intended to analyze and present data resembling clinical practice.

At baseline, patients with IGHD were demonstrated to be different entities and differ significantly from patients with MPHDs, especially for age, onset of GHD, underlying diagnosis, and, consequently, in the number of patients who underwent pituitary surgery. Such differences in patient characteristics are comparable to data published by the KIMS study group $(23,24)$. Metabolically, the investigated patients were overweight. The mean BMI was profoundly higher compared with the BMI of $24.4 \mathrm{~kg} / \mathrm{m}^{2}$ in a healthy Dutch cohort of 4070 men and women with a comparable mean age (25). By comparing all cardiovascular risk factors with a different Dutch cohort of 293 healthy subjects of similar age, we (data not shown) demonstrated a worse metabolic profile in our GH-deficient population (26). However, due to a narrow range of age in this cohort of healthy subjects, the data have to be interpreted with caution. Patients with IGHD had a less impaired metabolic profile than patients with MPHDs. The fact that the included subgroup of patients with MPHDs had a lower BMI than the remaining patients with MPHDs in the registry could only have led to an underestimation of this outcome. After adjustment for age, patients with MPHDs still had a higher triglyceride level, lower HDL cholesterol, and a larger waist circumference, the latter two being observed only in men. To investigate the influence of the heterogeneity of the two groups on the metabolic parameters, patients with adultonset GHD or with a non-functioning pituitary adenoma as an underlying cause of GHD were investigated. The difference in waist circumference was no longer present but the differences in the lipid profile persisted. The effects of other pituitary hormone deficiencies or their replacement therapies in patients with MPHDs might have had influence on the lipid profile before starting $\mathrm{GH}$ treatment. However, the KIMS study group did not find any metabolic differences between patients with IGHD and those with MPHDs. The differences encountered in our study could also be due to the fact that patients with IGHD are less GH deficient than patients with MPHDs, leading to a lesser detrimental effect of GHD on metabolic indices in IGHD (27). Nevertheless, adjusting for GH peak level (data not shown) did not affect the metabolic differences.

During follow-up, the BMI and waist circumference increased significantly in both groups, but significantly more in patients with IGHD compared with those having MPHDs. Short-term studies on GH treatment in patients with GHD demonstrated a positive effect on body composition (more prone in waist circumference than in BMI) (5). However, long-term studies, as reviewed recently by Appelman-Dijkstra et al. (28), have demonstrated an increase or no change in BMI, and mostly no change in waist circumference. The normal aging effect in long-term studies has to be taken into account. In a cohort study of Dutch healthy adults of the same mean age, an increase in the BMI of $1.6 \mathrm{~kg} / \mathrm{m}^{2}$ over 11 years of follow-up was demonstrated (25). During GH treatment, patients with IGHD gained more weight than patients with MPHDs. However, when analyzing only patients with a nonfunctioning pituitary adenoma, this difference was no longer present. Probably, the heterogeneity in underlying diagnosis is more important than the extension of hypopituitarism for the effect of $\mathrm{GH}$ treatment on body composition. GH treatment seemed to have a positive effect on lipid profile in patients with IGHD, except for triglycerides. These effects were less than that observed in patients with MPHDs. The positive effect of $\mathrm{GH}$ treatment on cholesterol, but not on triglycerides, has been previously demonstrated in studies with more than 5 years of follow-up (28). However, the use of lipidlowering drugs has not always been considered. After excluding patients using lipid-lowering medication from our study, the effect diminished but the positive effect on HDL cholesterol persisted. The lowering effect on total and LDL cholesterol was no longer significant but also did not increase, indicating a preventive role of $\mathrm{GH}$ treatment by inhibiting the expected increase in cholesterol level with age and (abdominal) body fat. Patients with MPHDs demonstrated a positive effect of $\mathrm{GH}$ treatment on triglyceride level, whereas patients with IGHD did not. This difference was most prevalent in women. A difference in the association of the GH-IGF1 axis with triglyceride level per gender has been reported previously (29). The increased level of GH-binding protein in women was 
thought to be a contributing factor, influencing the lipolytic activity of $\mathrm{GH}$ (29). The interaction of $\mathrm{GH}$ treatment with sex hormone levels or replacement therapy is a matter of debate (16). In our study, women with MPHDs could have experienced an interaction with estrogen replacement, inhibiting the lipolytic activity. On the other hand, women with IGHD received lower doses of GH than those with MPHDs, which could have diminished this difference in effect. The higher increase in waist circumference in the group of patients with IGHD might also have influenced the results of $\mathrm{GH}$ effect on triglyceride level leading to a lesser effect on IGHD. Subsequent to the increase in waist circumference in our cohort, HbA1c increased during long-term follow-up equally in both groups. One might have expected a greater increase in patients with IGHD due to a higher increase in waist circumference. However, patients (female) with IGHD received a lower dose of $\mathrm{GH}$, which might have led to a less detrimental effect on insulin sensitivity. In addition, in patients with MPHDs, a contributing negative effect of, for example, glucocorticoid replacement therapy on glucose metabolism has to be considered. After excluding patients using antihypertensive medication, systolic blood pressure did not change over time and diastolic blood pressure increased slightly. GH treatment might have protected against the aging effect on blood pressure in both groups equally. A Dutch cohort of healthy subjects of resembling age demonstrated a higher increase in diastolic blood pressure during 6 years of follow-up (0.8 mmHg/year) (26).

To date, only three studies have reported on the risk or incidence of CVDs in GH-treated adult patients with more than 2 years of follow-up $(9,10,11,30)$. The assumed increased risk for CVDs diminished and even a decreased risk for CVDs was found in one (11). In our study, the incidence of CVDs was $0.7 / 100$ patient-years. This number resembles the incidence in the study by Holmer et al. (10), which was comparable to matched Swedish controls. Despite the relatively short follow-up, the risk for CVDs in our study did not differ between patients with IGHD and those with MPHDs. The incidence of diabetes mellitus was similar to the incidence reported by Attanasio et al. (31) in a large international post-marketing database, and comparable to European reference data used. However, comparisons are difficult to interpret due to differences in definitions, date of data collection, and contributing factors such as BMI and physical activity. The risk of developing diabetes during GH treatment did not differ between patients with IGHD and those with MPHDs. The factors of age and BMI were important confounders.
Hypopituitarism is a dynamic condition. In our study population, $17 \%$ of patients with IGHD developed another pituitary hormone deficiency during GH treatment. Klose et al. (24) described an incidence of 35\% in 165 out of 283 patients with IGHD with available data. The lower percentage of patients developing a new deficiency in our cohort could be due to an overestimation in the KIMS cohort due to registration bias: only changes in medication were asked to be reported. Following that, there is also the risk of undiagnosed pituitary hormone deficiencies that might influence the results of this study. However, this could have been the case in both patient groups. A result could have been an underestimation of the effect of $\mathrm{GH}$ treatment. It could partly explain the increase in the BMI and waist circumference being more in patients with IGHD than those with MPHDs.

This is the second observational study on the effect of GH treatment in patients with IGHD compared with patients with MPHDs. We describe a significant longer follow-up period and due to a sophisticated method of longitudinal data analysis, we were able to properly deal with missing data, and therefore, loss of power was averted. Thorough sensitivity analyses were also performed. In our study, detailed registration of adverse events and medication use during follow-up was ensured via monitoring the medical records (bi-)annually with the help of trained nurses. This also made it possible to account for the use of cardioprotective medication, which is often overlooked. However, limitations have to be addressed. Owing to the observational nature of this study, we were unable to standardize diagnostic testing in all patients and hence undiagnosed pituitary hormone deficiencies could have existed. Following that, there is a diversity of incorporated laboratory methodologies that could influence between-group and within-individual analyses. The collection of blood samples for triglycerides in a fasting state could not be verified in all cases. However, the distribution of these influencing factors is at random; hence, the overall effect on the described results, at least for between-group analyses, will be small. However, we emphasize that our data merely represent clinical practice, and a prospective case-control study should be carried out to overcome these pitfalls. Another limitation is the lack of a control group of untreated GHD adults to correct for the aging effect and to draw firm conclusions purely on the effect of $\mathrm{GH}$ treatment. However, desired randomized controlled trials on longterm treatment are both impractical and unethical.

In conclusion, our results demonstrate that patients with IGHD have a less impaired metabolic profile than 
patients with MPHDs. Despite the aging-related weight gain, GH seems to protect patients with GHD against unfavorable lipid levels and blood pressure in both patients with IGHD and those with MPHDs. In clinical practice, patients with either entity deserve similar opinions by their attending endocrinologist to assess the benefit of GH replacement therapy.

\section{Declaration of interest}

The authors declare that there is no conflict of interest that could be perceived as prejudicing the impartiality of the research reported.

\section{Funding}

C C van Bunderen was supported by an AGIKO grant of The Netherlands Organisation for Health Research and Development (ZonMw) (grant number 92003591). Establishment of the Dutch National Registry of Growth Hormone Treatment in Adults was financially supported by the Health Care Insurance Board.

\section{Acknowledgements}

The authors are very grateful to all colleagues at the Internal and Endocrine departments of all participating Dutch hospitals for their collaboration.

\section{References}

1 de Boer H, Blok GJ \& Van der Veen EA. Clinical aspects of growth hormone deficiency in adults. Endocrine Reviews 199516 63-86. (doi:10.1210/er.16.1.63)

2 Cuneo RC, Salomon F, McGauley GA \& Sonksen PH. The growth hormone deficiency syndrome in adults. Clinical Endocrinology 199237 387-397. (doi:10.1111/j.1365-2265.1992.tb02347.x)

3 Rosèn T \& Bengtsson BA. Premature mortality due to cardiovascular disease in hypopituitarism. Lancet 1990336 285-288. (doi:10.1016/ 0140-6736(90)91812-O)

4 Stochholm K, Gravholt CH, Laursen T, Laurberg P, Andersen M, Kristensen LO, Feldt-Rasmussen U, Christiansen JS, Frydenberg M \& Green A. Mortality and GH deficiency: a nationwide study. European Journal of Endocrinology 2007 157 9-18. (doi:10.1530/EJE-07-0013)

5 Maison P, Griffin S, Nicoue-Beglah M, Haddad N, Balkau B \& Chanson P. Impact of growth hormone (GH) treatment on cardiovascular risk factors in GH-deficient adults: a Metaanalysis of Blinded, Randomized, Placebo-Controlled Trials. Journal of Clinical Endocrinology and Metabolism 200489 2192-2199. (doi:10.1210/jc.2003-030840)

6 Salomon F, Cuneo RC, Hesp R \& Sonksen PH. The effects of treatment with recombinant human growth hormone on body composition and metabolism in adults with growth hormone deficiency. New England Journal of Medicine 1989321 1797-1803. (doi:10.1056/ NEJM198912283212605)

7 Gibney J, Wallace JD, Spinks T, Schnorr L, Ranicar A, Cuneo RC, Lockhart S, Burnand KG, Salomon F, Sonksen PH et al. The effects of 10 years of recombinant human growth hormone $(\mathrm{GH})$ in adult GH-deficient patients. Journal of Clinical Endocrinology and Metabolism 199984 2596-2602. (doi:10.1210/jcem.84.8.5916)

8 Gotherstrom G, Bengtsson BA, Bosaeus I, Johannsson G \& Svensson J. A 10-year, prospective study of the metabolic effects of growth hormone replacement in adults. Journal of Clinical Endocrinology and Metabolism 200792 1442-1445. (doi:10.1210/jc.2006-1487)

9 Hartman ML, Xu R, Crowe BJ, Robison LL, Erfurth EM, Kleinberg DL, Zimmermann AG, Woodmansee WW, Cutler GB Jr, Chipman JJ et al. Prospective safety surveillance of GH-deficient adults: comparison of GH-treated vs untreated patients. Journal of Clinical Endocrinology and Metabolism 201398 980-988. (doi:10.1210/jc.2012-2684)

10 Holmer H, Svensson J, Rylander L, Johannsson G, Rosen T, Bengtsson BA, Thoren M, Hoybye C, Degerblad M, Bramnert M et al. Nonfatal stroke, cardiac disease, and diabetes mellitus in hypopituitary patients on hormone replacement including growth hormone. Journal of Clinical Endocrinology and Metabolism 200792 3560-3567. (doi:10.1210/jc.2007-0458)

11 Svensson J, Bengtsson BA, Rosen T, Oden A \& Johannsson G. Malignant disease and cardiovascular morbidity in hypopituitary adults with or without growth hormone replacement therapy. Journal of Clinical Endocrinology and Metabolism 200489 3306-3312. (doi:10.1210/jc. 2003-031601)

12 Erfurth EM. Update in mortality in GH-treated patients. Journal of Clinical Endocrinology and Metabolism 201398 4219-4226. (doi:10.1210/ jc.2013-2415)

13 van Bunderen CC, van Nieuwpoort IC, Arwert LI, Heymans MW, Franken AA, Koppeschaar HPF, van der Lely AJ \& Drent ML. Does growth hormone replacement therapy reduce mortality in adults with growth hormone deficiency? Data from the dutch national registry of growth hormone treatment in adults. Journal of Clinical Endocrinology and Metabolism 201196 3151-3159. (doi:10.1210/jc.2011-1215)

14 Burman P, Johansson AG, Siegbahn A, Vessby B \& Karlsson FA. Growth hormone (GH)-deficient men are more responsive to $\mathrm{GH}$ replacement therapy than women. Journal of Clinical Endocrinology and Metabolism 199782 550-555. (doi:10.1210/jcem.82.2.3776)

15 Hayes FJ, Fiad TM \& McKenna TJ. Gender difference in the response of growth hormone (GH)-deficient adults to GH therapy. Metabolism Clinical and Experimental 199948 308-313. (doi:10.1016/S00260495(99)90077-X)

16 Span JP, Pieters GF, Sweep CG, Hermus AR \& Smals AG. Gender difference in insulin-like growth factor I response to growth hormone $(\mathrm{GH})$ treatment in GH-deficient adults: role of sex hormone replacement. Journal of Clinical Endocrinology and Metabolism $2000 \mathbf{8 5}$ 1121-1125. (doi:10.1210/jcem.85.3.6463)

17 Gaillard RC, Mattsson AF, Akerblad AC, Bengtsson BA, Cara J, FeldtRasmussen U, Koltowska-Haggstrom M, Monson JP, Saller B, Wilton P et al. Overall and cause-specific mortality in GH-deficient adults on GH replacement. European Journal of Endocrinology 2012166 1069-1077. (doi:10.1530/EJE-11-1028)

18 Attanasio AF, Bates PC, Ho KK, Webb SM, Ross RJ, Strasburger CJ, Bouillon R, Crowe B, Selander K, Valle D et al. Human growth hormone replacement in adult hypopituitary patients: long-term effects on body composition and lipid status - 3-year results from the HypoCCS Database. Journal of Clinical Endocrinology and Metabolism 200287 1600-1606. (doi:10.1210/jcem.87.4.8429)

19 Gutierrez LP, Koltowska-Haggstrom M, Jonsson PJ, Mattsson AF, Svensson D, Westberg B \& Luger A. Registries as a tool in evidencebased medicine: example of KIMS (Pfizer International Metabolic Database). Pharmacoepidemiology and Drug Safety 2008 17 90-102. (doi:10.1002/pds.1510)

20 van Nieuwpoort IC, van Bunderen CC, Arwert LI, Franken AA, Koppeschaar HP, van der Lely AJ, Twisk J, Boers M \& Drent ML. Dutch National Registry of Growth Hormone Treatment in Adults: patient characteristics and diagnostic test procedures. European Journal of Endocrinology 2011164 491-497. (doi:10.1530/EJE-10-0914)

21 Consensus guidelines for the diagnosis and treatment of adults with growth hormone deficiency: summary statement of the Growth Hormone Research Society Workshop on Adult Growth Hormone Deficiency. Journal of Clinical Endocrinology and Metabolism $1998 \mathbf{8 3}$ 379-381. (doi:10.1210/jcem.83.2.4611) 
22 Fitzmaurice GM, Laird NM, Ware JH. In Applied Longitudinal Analysis, 2nd edn, Hoboken, New Jersey: John Wiley \& Sons, Inc., 2011.

23 Abs R, Mattsson AF, Bengtsson BA, Feldt-Rasmussen U, Goth MI, Koltowska-Haggstrom M, Monson JP, Verhelst J \& Wilton P. Isolated growth hormone (GH) deficiency in adult patients: baseline clinical characteristics and responses to GH replacement in comparison with hypopituitary patients. A sub-analysis of the KIMS database. Growth Hormone \& IGF Research 200515 349-359. (doi:10.1016/j.ghir.2005.06.018)

24 Klose M, Jonsson B, Abs R, Popovic V, Koltowska-Haggstrom M, Saller B, Feldt-Rasmussen U \& Kourides I. From isolated GH deficiency to multiple pituitary hormone deficiency: an evolving continuum - a KIMS analysis. European Journal of Endocrinology 2009161 (Suppl 1) S75-S83. (doi:10.1530/EJE-09-0328)

25 Nooyens AC, Visscher TL, Verschuren WM, Schuit AJ, Boshuizen HC, van Mechelen W \& Seidell JC. Age, period and cohort effects on body weight and body mass index in adults: The Doetinchem Cohort Study. Public Health Nutrition 200912 862-870. (doi:10.1017/ S1368980008003091)

26 van Bussel BC, Schouten F, Henry RM, Schalkwijk CG, de Boer MR, Ferreira I, Smulders YM, Twisk JW \& Stehouwer CD. Endothelial dysfunction and low-grade inflammation are associated with greater arterial stiffness over a 6-year period. Hypertension 201158 588-595. (doi:10.1161/HYPERTENSIONAHA.111.174557)
27 Toogood AA, Beardwell CG \& Shalet SM. The severity of growth hormone deficiency in adults with pituitary disease is related to the degree of hypopituitarism. Clinical Endocrinology 199441 511-516. (doi:10.1111/j.1365-2265.1994.tb02583.x)

28 Appelman-Dijkstra NM, Claessen KM, Roelfsema F, Pereira AM \& Biermasz NR. Therapy of Endocrine disease: long-term effects of recombinant human GH replacement in adults with GH deficiency: a systematic review. European Journal of Endocrinology 2013169 R1-R14. (doi:10.1530/EJE-12-1088)

29 van Bunderen CC, Oosterwerff MM, van Schoor NM, Deeg DJH, Lips P \& Drent ML. Serum IGF1, metabolic syndrome, and incident cardiovascular disease in older people: a population-based study. European Journal of Endocrinology 2013168 393-401. (doi:10.1530/EJE-12-0784)

30 Gazzaruso C, Gola M, Karamouzis I, Giubbini R \& Giustina A. Cardiovascular risk in adult patients with growth hormone (GH) deficiency and following substitution with GH - an update. Journal of Clinical Endocrinology and Metabolism 201499 18-29. (doi:10.1210/jc. 2013-2394)

31 Attanasio AF, Jung H, Mo D, Chanson P, Bouillon R, Ho KK, Lamberts SW \& Clemmons DR. Prevalence and incidence of diabetes mellitus in adult patients on growth hormone replacement for growth hormone deficiency: a surveillance database analysis. Journal of Clinical Endocrinology and Metabolism 201196 2255-2261. (doi:10.1210/jc. 2011-0448)

Received 24 January 2014

Revised version received 28 April 2014

Accepted 1 May 2014 